\title{
Stator Design and Performance of Superconducting Motors for Aerospace Electric Propulsion Systems
}

\author{
Charalampos D. Manolopoulos, Matteo F. Iacchetti, Member, IEEE, Alexander C. Smith, Senior Member, IEEE, Kévin Berger,
} Mark Husband, and Paul Miller

\begin{abstract}
Hybrid electric propulsion has been identified as a potential solution to the ambitious environmental emissions and noise targets of the aerospace industry. Superconducting machines may be the key component of that topology enabling the high power densities and efficiencies needed in aerospace. Fully superconducting machines, however, are not a mature technology. This paper looks at the different machine design configurations focusing on the stator magnetic circuit of a fully superconducting motor. The motor has been designed for an aerospace distributed fan propulsion motor with an aerospace benchmark specification of $1 \mathrm{MW}$. The AC fully superconducting machine includes superconducting bulk magnets mounted on a conventional rotor core and an $\mathbf{M g B}_{2}$ superconducting wire wound stator. The AC losses in the stator winding are particularly sensitive to exposure to the main rotor field so different screening solutions were used to shield the superconducting windings from the rotor field. The effectiveness of the screening techniques for the stator coils and the impact on the machine performance and weight were evaluated for different stator designs, such as full stator core and air core with and without flux diverters. Various combinations of pole numbers, diverter geometries and magnetic materials have been checked. Results show that there is a tradeoff between stator iron losses and superconducting losses.
\end{abstract}

Index Terms-Superconducting AC machine, Flux diverter, AC superconducting losses, Magnesium Diboride.

\section{INTRODUCTION}

A IR TRAFFIC growth and environmental regulations to reduce noise and emissions are driving major improvements in aerospace fuel and noise efficiencies. One of the disruptive technologies identified as promising is the hybrid electric distributed propulsion (HEDP) accompanied by a redesign of the airframe such as the hybrid wing body [1], [2]. Whilst conventional electrical power systems may be used for smaller

Manuscript receipt and acceptance dates will be inserted here. This work was supported in part by the EPSRC, U.K. and in part by Rolls-Royce plc.

C. D. Manolopoulos, M. F. Iacchetti and A. C. Smith are with the Power and Energy Division, School of Electrical and Electronic Engineering, The University of Manchester, Manchester M13 9PL, U.K. (e-mail: charalampos.manolopoulos@ postgrad.manchester.ac.uk;

matteo.iacchetti@manchester.ac.uk; sandy.smith@manchester.ac.uk).

$\mathrm{K}$. Berger is with the Group of Research in Electrical Engineering of Nancy, Faculties of Sciences and Technologies, University of Lorraine, 54506 Vandoeuvre-lès-Nancy, France (e-mail: kevin.berger@univ-lorraine.fr).

M. Husband is with the Electrical Capability Group, Rolls-Royce plc, Derby DE24 8BJ, U.K. (e-mail: mark.husband@rolls-royce.com).

P. Miller is with the Rolls-Royce Future Technologies Group, Derby DE24 8BJ, U.K. (e-mail: paul.miller3@rolls-royce.com).

Color versions of one or more of the figures in this paper are available online at http://ieeexplore.ieee.org.

Digital Object Identifier will be inserted here upon acceptance. aircraft, much larger aircraft are likely to require the use of superconducting electrical power systems to enable the required whole system power density and efficiency levels to be achieved. HEDP consists of distributed electric fan propulsion units connected to a number of generator/gas units through power management units. In these systems the gas-turbine is decoupled from propulsion units and studies have shown that this reduces emissions and noise [3], [4]. A power dense and high efficiency superconducting motor is the key component of this promising architecture and the development of such a machine designed for aircraft propulsion systems would enable the hybrid electric aero-propulsion concept [5], [6].

Early superconducting machines were generally large synchronous generators using superconducting dc field windings [7]. However to meet the high efficiencies and power densities of aerospace, fully superconducting machines were considered. Fully superconducting machines offer higher electrical loadings than the partially superconducting machines. Another advantage is the reduced airgap which increases the magnetic loading as the stator armature and the rotor dc winding have similar temperatures. Different fully superconducting machine prototypes have been developed worldwide to demonstrate the feasibility of the concept. A fully superconducting machine for an electric vehicle [8] and an inductor type fully superconducting machine for ship propulsion [9] were built in Japan. A fully superconducting prototype with YBCO stator coils operating in liquid nitrogen bath was developed in Cambridge, UK [10] and a fully superconducting machine cooled by iron conduction was built in China [11]. Fully superconducting machines have also been proposed for offshore wind turbine applications [12].

The major challenge of fully superconducting machines is the increased AC losses in the stator windings. AC losses are an important consideration because high losses in the superconducting stator windings could diminish the benefits of a fully superconducting design by requiring large cooling systems. AC losses in superconducting coils or tapes have been studied mainly in small samples or coils [13], [14]. Flux diverters have also been examined to reduce the overall losses but most of the studies have focused on transport applications [15], [16] or sinusoidal external fields [17]. That is not the case in a machine environment where the field seen by the stator conductors cannot be considered as purely alternating.

This paper looks at different stator design configurations of a fully superconducting motor for an aerospace distributed fan motor. The machine performance in terms of weight and effi- 


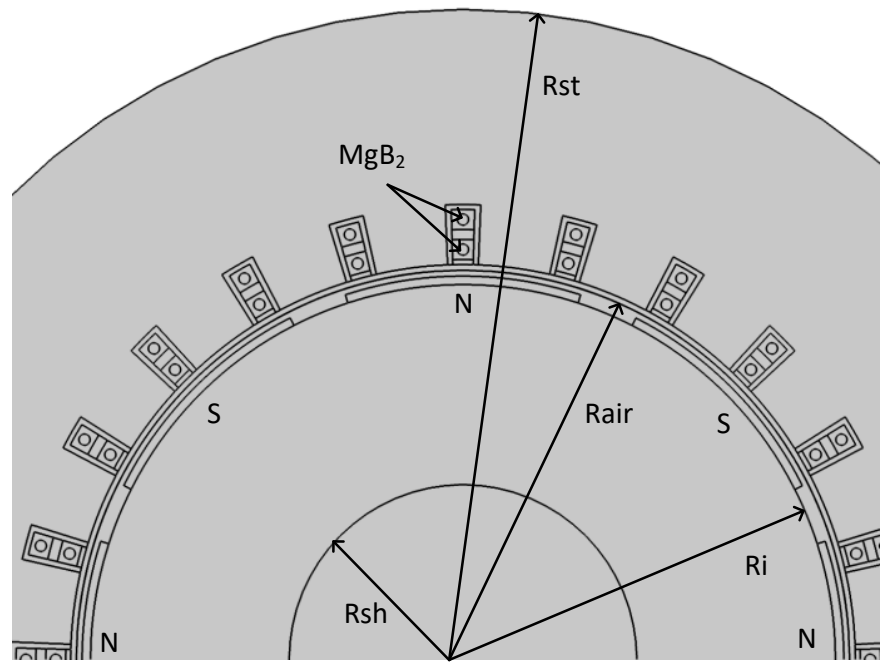

Fig. 1. FE model of $1 \mathrm{MW}$ AC superconducting machine.

TABLE I

DESIGN PARAMETERS OF THE AC SUPERCONDUCTING MACHINE

\begin{tabular}{lclc}
\hline \multicolumn{1}{c}{ Quantity } & Value & \multicolumn{1}{c}{ Quantity } & Value \\
\hline Machine power & $1 \mathrm{MW}$ & Number of slots & 24 \\
Mechanical speed & $12,000 \mathrm{rpm}$ & Axial length & $0.3 \mathrm{~m}$ \\
Pole pairs & 4 & Shaft radius $R s h$ & $0.07 \mathrm{~m}$ \\
Rated Torque & $800 \mathrm{Nm}$ & Rotor iron radius $R i$ & $0.15 \mathrm{~m}$ \\
Phase voltage & $800 \mathrm{Vrms}$ & Airgap radius Rair & $0.1557 \mathrm{~m}$ \\
Phase current & $560 \mathrm{Arms}$ & Outer radius Rst & $0.26 \mathrm{~m}$ \\
Current per coil $^{\mathrm{a}}$ & $140 \mathrm{Arms}$ & Turns per coil & 5 \\
\hline \hline
\end{tabular}

aThe rated emf is achieved by using 5 turns per stator coil, with two coils in series and four groups in parallel.

TABLE II

SPECIFICATIONS OF THE MGB 2 WIRE USED IN ARMATURE

\begin{tabular}{lc}
\hline \hline Manufacturer & HyperTech Inc. \\
Superconducting Material & Mono-core $\mathrm{MgB}_{2}$ \\
Critical current density $(20 \mathrm{~K}, 1.37 \mathrm{~T})$ & $1.5 \mathrm{kA} / \mathrm{mm}^{2}$ \\
Voltage criterion $E_{0}$ & $1 \mu \mathrm{V} / \mathrm{cm}$ \\
Index of power law $n$ & 30 \\
Sheath material & Stainless Steel \\
Resistivity of sheath & $540 \mathrm{n} \Omega \mathrm{m}$ \\
Filament diameter & $0.18 \mathrm{~mm}$ \\
Total diameter & $0.36 \mathrm{~mm}$ \\
\hline \hline
\end{tabular}

ciency was evaluated for the different stator designs.

\section{SuPerconducting Machine Design}

A suitable benchmark motor specification has been chosen for a distributed electric fan motor for a short-haul aircraft. These commonly have a gearbox so that a high-speed motor can be used to reduce weight and improve the power density. The fan motor is assumed here to have an output power of $1 \mathrm{MW}$ with a maximum speed of $12,000 \mathrm{rpm}$. The design guidelines for a permanent-magnet (PM) machine were initially used to meet the specifications. Fig. 1 shows the basic dimensions of the motor which are given in Table I along with the basic design parameters. Similar to the AC PM machine, the superconducting machine will have 8-poles, which is typical for this type of machine. It is also assumed that a maximum phase voltage of $800 \mathrm{~V}_{\text {rms }}$ is available. This voltage would currently be regarded as a little high (line-line

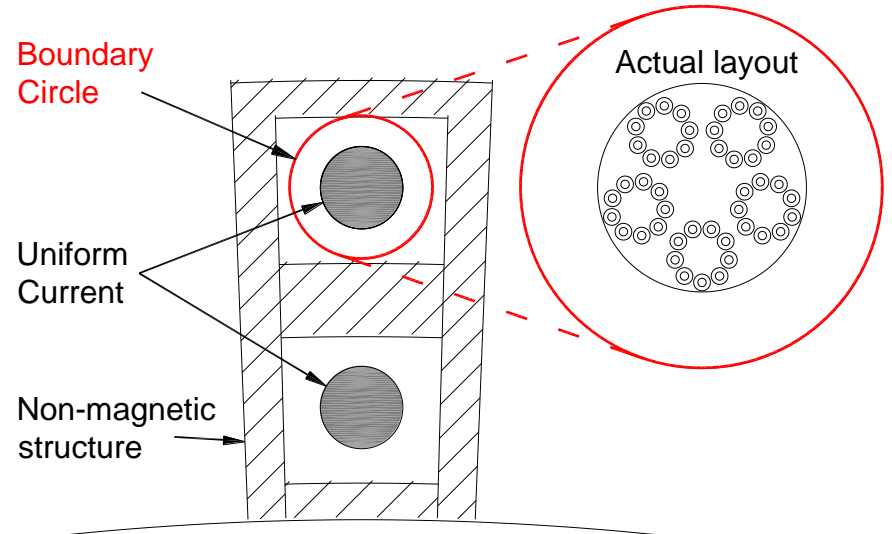

Fig. 2. Illustration of a slot for the air-core stator design. The tangential field at the boundary circle is used to couple the machine model with the superconducting wire model.

volts $>1000 \mathrm{~V}$ ) because of potential breakdown at altitude however, it was chosen here to keep the current levels down. There are on-going studies looking at increasing electrical system voltage levels in aerospace applications.

The rotor design was not considered here. A detailed rotor design would determine the airgap and the geometry that ensure both the required magnetic loading and protection from demagnetization. The rotor was modelled using the same rotor model as a PM machine shown in Fig. 1. Conventional PM blocks with unity recoil permeability were used. Only the 'equivalent' remanent flux density of the blocks was varied to represent the high fields produced by the superconducting bulks [10], [18] or stacks of superconducting tapes [19].

The stator AC winding was modelled using Hyper Tech $0.36 \mathrm{~mm}$ mono-core round wire. Table II gives the specifications of the wire. This wire was originally designed for superconducting fault current limiters, so it has a simple high resistance steel sheath around the $\mathrm{MgB}_{2}$ core. Although not ideally optimal for this particular application, it has previously demonstrated very good low-loss and consistent performance under AC excitation.

A remanent flux density of approximately $14 \mathrm{~T}$ would be required to provide the rated back-emf and rated output power with a non-magnetic stator. For this design the maximum external rotor field seen by the stator conductors was estimated at $1.37 \mathrm{~T}$. The estimated peak critical current density for this external magnetic field and an assumed operating temperature of $20 \mathrm{~K}$ was $1.5 \mathrm{kA} / \mathrm{mm} 2$. The wire fill factor was $25 \%$ giving an estimated critical current for this wire of $38.2 \mathrm{~A}$. The rated current for the machine is $140 \mathrm{~A}_{\mathrm{rms}}$, so 9 strands of $0.36 \mathrm{~mm}$ $\mathrm{MgB}_{2}$ wire were used with each strand operating at approximately $58 \%$ of the critical current. This would seem to be rather low but the machine was assumed to have an overload capacity of 50\%, which would increase the operating current on overload to just under $90 \%$ of the critical current.

\section{Estimation OF AC SUPERCONDUCTING LOSSES}

Two models were indirectly coupled to estimate the AC superconducting losses as shown in Fig. 2. The first machine model uses uniform current density to calculate the field dis- 


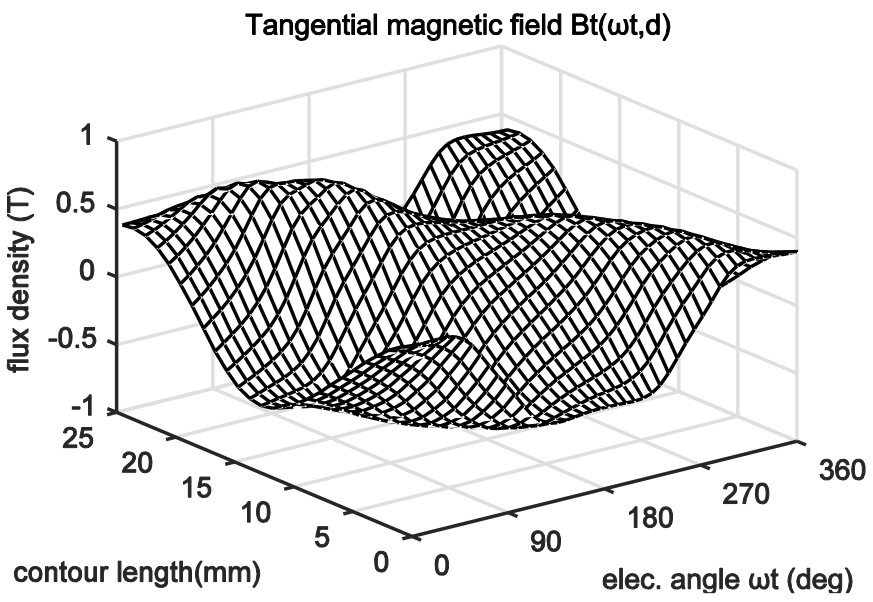

Fig. 3. Tangential magnetic flux density $B t(\omega t, d)$ along the boundary contour for one electrical cycle for an outer layer coil of the air-core stator.

tribution, neglecting the superconducting properties. The stator current in the machine model is aligned with the q-axis of the rotor to achieve maximum torque. The tangential magnetic field at the boundary circle is then applied to the second superconducting wire model to obtain the current distribution and finally the superconducting losses [20], [21]. Fig. 3 shows the tangential field around the boundary circle used to couple the two models for an outer layer coil of the air-cored stator.

The 2D FE superconducting wire model uses the $\mathrm{H}$-formulation to simulate the cross section of the real arrangement of the superconducting coils [22], [23]. The governing equation is:

$$
\nabla \times(\rho \nabla \times \mathbf{H})+\frac{\partial(\mu \mathbf{H})}{\partial t}=0
$$

where $\rho, \mu$, and $\mathbf{H}$ are the resistivity, the permeability and the magnetic field, respectively. The sheath of the superconducting wire does not include any magnetic material so a constant permeability is used, $\mu=\mu_{0}$. The resistivity of the sheath is constant whereas for the $\mathrm{MgB}_{2}$ the non-linear power law was used: $\rho_{s c}=\left(E_{0} / J_{c}\right)\left(J / J_{c}\right)^{n-1}$. Generally, $J_{c}$ varies with temperature and magnetic field; however a constant critical current model was used to reduce the simulation time. The sheath resistivity, the constant critical current density and the values of $E_{0}$ and $n$ are given in Table II.

The superconductor wire losses can be attributed to several factors: hysteretic losses in the $\mathrm{MgB}_{2}$; eddy current losses in the sheath material; coupling losses between the strands of $\mathrm{MgB}_{2}$ and proximity losses which are a redistribution of the eddy currents caused by the proximity of different currentcarrying conductors. The eddy current losses in the sheath are negligible due to the high resistivity of the stainless steel. Transposition is assumed to eliminate the coupling losses. Each strand carries the same current even if the strands are connected at the coil ends.

Fig. 4 shows the current density distribution for an outer-layer coil of the air-cored stator. The proximity losses can

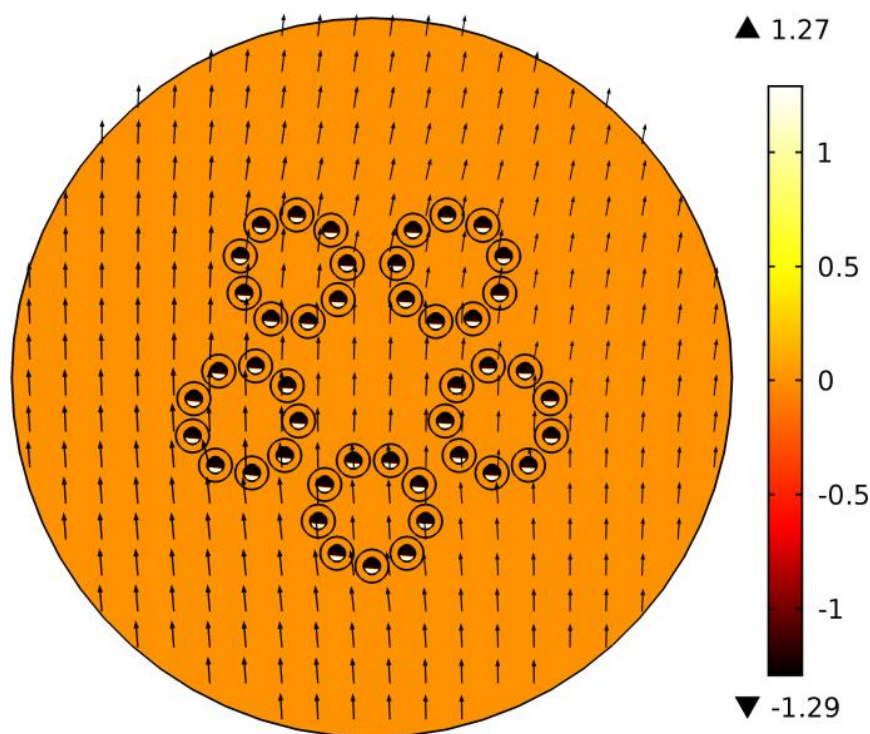

Fig. 4. Normalized current density distribution $J / J_{c}$ in an outer-layer coil of the air-core stator after one electrical period (north magnet below the slot). The coil has 5 turns composed of 9 strands. Each strand carries the same current as transposition is assumed.
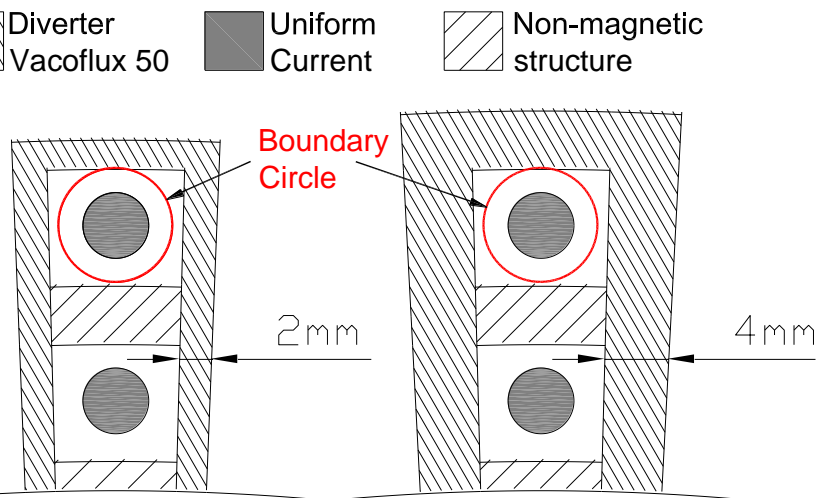

Fig. 5. Schematic diagram of thin (left) and thick (right) flux diverter position around the superconducting coils.

be neglected for an air-cored stator as the major loss factor is the magnetization losses in the superconductors due to the exposure from the main rotor field. Fig. 4 shows the eddy currents produced inside each superconducting strand by the external rotating magnetic field. These eddy currents are responsible for the significant increase in the losses compared to the self-field transport losses.

\section{DifFerent Machine Designs}

\section{A. Flux Diverter Design}

To reduce the field seen by the superconducting coils a C-shape flux diverter was initially proposed and tested. A flux diverter was placed around the two coils of each slot with the open side towards the rotor, as shown in Fig. 5. This design allows the flux to pass around the coils and at the same time reduces the flux passing through the superconducting coils. In order to assess the different designs the magnetic loading was assumed to be constant. The first harmonic therefore of the magnetic flux density in the airgap was maintained at $1 \mathrm{~T}$ by 


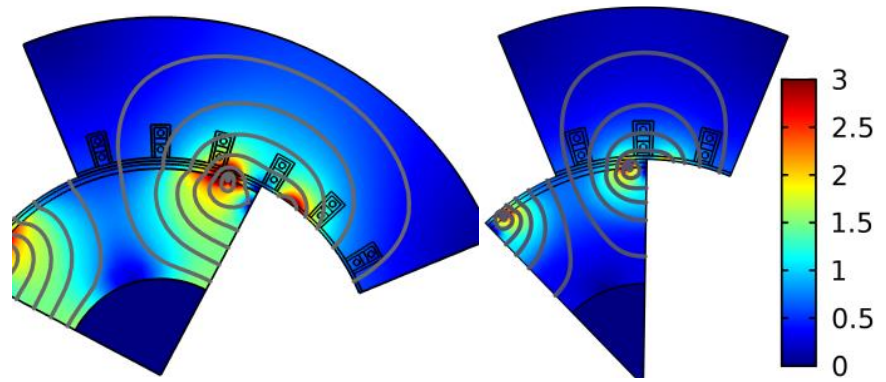

Fig. 6. Magnetic field distribution for a single pole for the 4-pole (left) and the 8-pole (right) air-core design. The flux path for the 4-pole design is considerably longer compare to the 8-pole design.

TABLE III

STATOR SuPERCONDUCTING LOSSES FOR DIFFERENT MACHINE DESIGNS

\begin{tabular}{lcccccc}
\hline \hline \multicolumn{1}{c}{\begin{tabular}{c} 
Superconducting \\
\multicolumn{1}{c}{ losses $^{1}$}
\end{tabular}} & \multicolumn{2}{c}{ 4-Pole Designs } & \multicolumn{4}{c}{ 8-Pole Designs } \\
& $\mathrm{AC}_{4}$ & $\mathrm{FD}_{4}$ & $\mathrm{AC}_{8}$ & $\mathrm{FD}_{8}$ & $\mathrm{TFD}_{8}$ & $\mathrm{MS}_{8}$ \\
\hline Outer layer coil (W/m) & 259 & 239 & 269 & 196 & 161 & 13 \\
Inner layer coil (W/m) & 665 & 569 & 478 & 404 & 290 & 43 \\
$\begin{array}{l}\text { Total stator loss } \\
\text { (active length) (W) }\end{array}$ & 6653 & 5818 & 5378 & 4320 & 3247 & 403 \\
\hline \hline
\end{tabular}

Key: AC-Air core, FD-Flux diverter (2mm), TFD-Thick flux diverter (4mm), MS-magnetic stator, the subscript is the pole number.

${ }^{1}$ All the designs have $1 \mathrm{~T}$ magnetic loading and the same electrical loading.

changing the remanent flux density of the superconducting rotor magnets. A magnetic loading of $1 \mathrm{~T}$ is common in conventional PM machines but for a non-magnetic stator this value would only be achievable with strong superconducting magnets. The electric loading was also kept the same for all the different designs by setting the rated value of $140 \mathrm{~A}_{\mathrm{rms}}$ in each coil.

The criteria used to assess the diverter performance and generally the stator design were the screening effectiveness, the iron losses and the added weight. The screening effectiveness was determined by calculating the average flux density inside the two coils of one slot for each rotor position during one electrical cycle. This value was compared with the corresponding one for an air-core stator with no flux diverters. Cobalt iron electrical steel Vacoflux50 from Vacuumschmelze $\mathrm{GmbH}$ was chosen for the flux diverters because of its high saturation flux density. Initially, thin diverters $2 \mathrm{~mm}$ thick were selected to avoid increasing the weight. This value was later doubled to assess the effect of diverter thickness on the machine performance.

\section{B. Pole Number Assessment - Four Pole Design}

Another way to reduce the superconducting losses is to reduce the frequency. This reduces both hysteresis losses in the superconducting coils and iron losses in the diverters. The frequency and pole number were reduced by half to maintain the high speed and power density. The geometry, the slot number and the current in each coil are the same as the 8-pole designs. To enable direct comparison of the designs, the magnetic loading was kept the same. The 4-pole air-core design requires higher-field superconducting magnets compared to the 8-pole air-core design because of the longer flux paths through the air, as shown in Fig. 6. The flux diverter performance was then tested in the 4-pole design.
TABLE IV

STATOR EFFICIENCY AND WEIGHT FOR DIFFERENT MACHINE DESIGNS

\begin{tabular}{lcccccc}
\hline \hline \multirow{2}{*}{ Design Parameter ${ }^{1}$} & 4-Pole Designs & \multicolumn{4}{c}{ 8-Pole Designs } \\
& $\mathrm{AC}_{4}$ & $\mathrm{FD}_{4}$ & $\mathrm{AC}_{8}$ & $\mathrm{FD}_{8}$ & $\mathrm{TFD}_{8}$ & $\mathrm{MS}_{8}$ \\
\hline Output Power (kW) & 925.9 & 925.9 & 850.7 & 909.8 & 967.6 & 1173.7 \\
Mean torque (Nm) & 736 & 736 & 677 & 724 & 770 & 934 \\
Torque ripple (\%) & 41 & 150 & 14 & 336 & 430 & 26 \\
Remanent Flux (T) & 27.5 & 25 & 11.5 & 10 & 8.2 & 2 \\
Screening (\%) & 0 & 21.4 & 0 & 30.8 & 54.4 & 87.5 \\
St. iron loss (W) & 0 & 1161 & 0 & 3391 & 5598 & 7037 \\
St. MgB ${ }_{2}$ loss (W) & 6653 & 5818 & 5378 & 4320 & 3247 & 403 \\
St. 'cold' inefficien- & 0.72 & 0.63 & 0.63 & 0.47 & 0.33 & 0.034 \\
cy' (\%) & & & & & & \\
St. efficiency (\%) & 99.29 & 99.25 & 99.37 & 99.16 & 99.09 & 99.37 \\
St. weight (kg) & 0.5 & 7.7 & 0.5 & 7.7 & 16 & 308 \\
\hline \hline
\end{tabular}

Key: AC-Air core, FD-Flux diverter (2mm), TFD-Thick flux diverter (4mm), MS-magnetic stator, the subscript is the pole number, St-Stator active length. ${ }^{1}$ All the designs have $1 \mathrm{~T}$ magnetic loading and the same electrical loading. ${ }^{2}$ This is defined as the armature superconducting losses in the cryogenic system as a percentage of the machine output power [6].

\section{RESULTS}

Table III gives the superconducting losses in each layer of a slot for one electrical cycle. It is assumed that each slot has the same losses so the total armature losses are the sum of the slot losses multiplied by the slot number and the active length. The end-winding losses are not included because the calculation of the field around the end windings would require a full 3D machine model. The end winding conductors would not be exposed to full rotor field so it is thought that the losses would be closer to the self-field losses. A more accurate estimation of the superconducting losses would require the calculation of the losses in each slot of a pole pitch due to the different phase angle of the field with respect to the coil current. Analytical models have shown that the losses are maximised when the current and magnetic field are in phase for low external magnetic fields with $H_{0}<<I_{d}(2 a \pi)$ for $2 a$ width strip [24]; however for higher external magnetic fields the losses are maximized at intermediate phase angles [14], [25]. The inner layer coil which is closer to the rotor has higher superconducting losses compared to the outer layer coil for all the designs because it is exposed to higher external fields. If the thickness of the flux diverters is increased, the losses are reduced, however the full magnetic stator has the lowest superconducting losses because the coils are not exposed to the full rotor field.

Table IV summarizes the performance figures for the different machine designs. The different magnetic circuit of each stator design would affect the rotor design and weight. Nevertheless, at this stage the rotor was kept the same for all the designs and only the remanent flux density of the magnets was changed. On one hand, as the volume of the iron is increased in the machine, the more effective the screening becomes and the lower the superconducting losses are. On the other hand, because of the extra mass, the added iron reduces the power density significantly. Direct comparison is possible here because the magnetic and electric loadings are the same for all the designs. In the 4-pole designs, higher-field superconducting magnets are needed compared to the 8-pole designs represented by the higher remanent flux densities in Table IV. The 
frequency is halved in the 4-pole designs compared to the 8pole designs. The 4-pole designs however produce higher superconducting losses because of the high fields at the coils.

For the 8-pole machine, the air-core design has a similar stator efficiency with the full magnetic stator design because the added iron losses are equal to the reduction of the superconducting losses due to screening. It should be noted however, that the superconducting losses are generated at $20 \mathrm{~K}$ so an air-core design, although very light, would impose a significant load on the cryocoolers. For an iron-cored machine however, the iron could be operated at room temperature but that would cause very high temperature gradients across the insulation between the coils and the iron. The flux diverters reduce the superconducting losses compared to the air-core design without adding significant weight independently of the pole number. However, the reduction is not significant and the superconducting losses remain prohibitive for the low operating temperature. Another problem with the flux diverter designs is the high torque ripple produced because of the non-uniform magnetic circuit (cogging torques). Different magnetic materials, for example soft magnetic composites (SMC), were also explored to reduce the weight of the diverters, but with no advantage. In these materials, the permeability drops quickly deteriorating the screening performance. The only design with manageable superconducting losses for the cryocoolers is the full magnetic stator design, which has a 'cold inefficiency' figure lower than $0.05 \%$ as indicated in [6, Table III]; The coefficient of performance is $1.46 \%$ [6], [12], assuming the cryocooler efficiency is $15 \%$ of Carnot efficiency and the ambient temperature at high altitude is $225 \mathrm{~K}$. So a motor dedicated cryocooler would require $28 \mathrm{~kW}$ at $225 \mathrm{~K}$ to remove the stator superconducting losses and the total stator efficiency would drop to $97 \%$. However, in order to increase the cryocooler efficiency and reduce the weight a centralised system with large cryocoolers and a liquid methane cryo-tank may be used [6]. Although the magnetic stator design has the lowest $\mathrm{MgB}_{2}$ losses, it suffers from high iron losses and weight. Further research on superconducting materials and/or winding techniques that reduce the sensitivity of the $\mathrm{AC}$ losses to external fields is required to enable a fully superconducting machine concept for hybrid electric aero-propulsion.

\section{CONCLUSIONS}

This paper looks at the design feasibility of the stator of a fully superconducting motor for an aerospace distributed fan motor. The selected fan motor was assumed to have an output power of $1 \mathrm{MW}$ with a maximum speed of $12,000 \mathrm{rpm}$. The $\mathrm{AC}$ superconducting machine used superconducting bulk magnets mounted on the rotor and a round $\mathrm{MgB}_{2}$ superconducting wire for the stator winding. The paper demonstrates a way of coupling the machine model with the superconducting wire model. This model is based on the real arrangement of the superconducting strands. The superconducting losses were calculated for different stator designs such as a full stator core and an air core with and without flux diverters. The effect of pole number and diverter thickness was examined as well. The results showed that there is a trade-off between added iron losses and the superconducting losses.

\section{REFERENCES}

[1] H. D. Kim, G. V. Brown, and J. L. Felder, "Distributed turboelectric propulsion for hybrid wing body aircraft," in 9th International Powered Lift conference, London, UK, 2008

[2] J. L. Felder, et al., "Turboelectric distributed propulsion in a hybrid wing body aircraft," in 20th ISABE conference, Gothenburg, Sweden, 2011.

[3] F. Berg, et al., "HTS Electrical System for a Distributed Propulsion Aircraft," IEEE Trans. Appl. Supercond., vol. 25, Jun 2015.

[4] P. Malkin and M. Pagonis, "Superconducting electric power systems for hybrid electric aircraft," Aircraft Engineering and Aerospace Technology, vol. 86, pp. 515-518, 2014.

[5] C. A. Luongo, et al., "Next Generation More-Electric Aircraft: A Potential Application for HTS Superconductors," IEEE Trans. Appl. Supercond., vol. 19, pp. 1055-1068, Jun 2009.

[6] F. Berg, et al., "HTS System and Component Targets for a Distributed Aircraft Propulsion System," IEEE Trans. Appl. Supercond., vol. 27, Jun 2017.

[7] S. S. Kalsi, et al., "Development status of rotating machines employing superconducting field windings," Proceedings of the IEEE, vol. 92, pp. 1688-1704, Oct 2004

[8] D. Sekiguchi, et al., "Trial Test of Fully HTS Induction/Synchronous Machine for Next Generation Electric Vehicle," IEEE Trans. Appl. Supercond., vol. 22, Jun 2012.

[9] T. Takeda, H. Togawa, and T. Oota, "Development of liquid nitrogencooled full superconducting motor," IHI Eng. Rev., vol. 39, p. 89, 2006.

[10] Z. Huang, et al., "Trial Test of a Bulk-Type Fully HTS Synchronous Motor," IEEE Trans. Appl. Supercond., vol. 24, Jun 2014.

[11] T. M. Qu, et al., "Development and testing of a $2.5 \mathrm{~kW}$ synchronous generator with a high temperature superconducting stator and permanent magnet rotor," Supercond. Sci. Technol., vol. 27, Apr 2014.

[12] X. Song, et al., "Design Study of Fully Superconducting Wind Turbine Generators," IEEE Trans. Appl. Supercond., vol. 25, pp. 1-5, 2015.

[13] X. Z. Pei, et al., "Design, Build and Test of an AC Coil Using MgB2 Wire for Use in a Superconducting Machine," IEEE Trans. Appl. Supercond., vol. 23, Jun 2013.

[14] F. Grilli, et al., "Computation of Losses in HTS Under the Action of Varying Magnetic Fields and Currents," IEEE Trans. Appl. Supercond., vol. 24, Feb 2014

[15] M. D. Ainslie, W. J. Yuan, and T. J. Flack, "Numerical Analysis of AC Loss Reduction in HTS Superconducting Coils Using Magnetic Materials to Divert Flux," IEEE Trans. Appl. Supercond., vol. 23, pp. 4700104-4700104, Jun 2013.

[16] D. Hu, et al., "Transport AC Loss Measurements of a Triangular EpoxyImpregnated High-Temperature Superconducting Coil," IEEE Trans. Appl. Supercond., vol. 27, Jun 2017.

[17] P. Kruger, et al., "Superconductor/ferromagnet heterostructures exhibit potential for significant reduction of hysteretic losses," Appl. Phys. Lett., vol. 102, May 202013.

[18] K. Berger, et al., "Magnetization and Demagnetization Studies of an HTS Bulk in an Iron Core," IEEE Trans. Appl. Supercond., vol. 26, Jun 2016.

[19] M. Baghdadi, et al., "Investigation of Demagnetization in HTS Stacked Tapes Implemented in Electric Machines as a Result of Crossed Magnetic Field," IEEE Trans. Appl. Supercond., vol. 25, Jun 2015

[20] V. M. R. Zermeno, et al., "Simulation of an HTS Synchronous Superconducting Generator," Superconductivity Centennial Conference 2011, vol. 36, pp. 786-790, 2012.

[21] X. Song, et al., "AC Losses and Their Thermal Effect in HighTemperature Superconducting Machines," IEEE Trans. Appl. Supercond., vol. 26, pp. 1-5, 2016.

[22] Z. Hong, A. M. Campbell, and T. A. Coombs, "Numerical solution of critical state in superconductivity by finite element software," Supercond. Sci. Technol., vol. 19, pp. 1246-1252, Dec 2006.

[23] R. Brambilla, F. Grilli, and L. Martini, "Development of an edgeelement model for AC loss computation of high-temperature superconductors," Supercond. Sci. Technol., vol. 20, pp. 16-24, 2007.

[24] Y. Mawatari and K. Kajikawa, "Hysteretic ac loss of superconducting strips simultaneously exposed to ac transport current and phase-different ac magnetic field," Appl. Phys. Lett., vol. 90, p. 022506, 2007.

[25] D. N. Nguyen, et al., "Experimental and numerical studies of the effect of phase difference between transport current and perpendicular applied magnetic field on total ac loss in Ag-sheathed (Bi, Pb) $2 \mathrm{Sr} 2 \mathrm{Ca} 2 \mathrm{Cu} 3$ O x tape," J. Appl. Phys., vol. 98, p. 073902, 2005. 